\title{
Pitfalls in the assessment of MGMT expression and in its correlation with survival in diffuse astrocytomas: proposal of a feasible immunohistochemical approach
}

Capper, David ; Mittelbronn, Michel ; Meyermann, Richard ; Schittenhelm, Jens

\begin{abstract}
Immunohistochemical studies showed that O6-methylguanine-DNA methyltransferase (MGMT) protein expression is negatively associated with survival in glioblastomas treated with alkylating agents in accordance with previous results of methylation-specific PCR. Implementation of this data in routine clinical diagnostics is limited due to often inappropriate study designs, e.g. pooling of tumor entities, WHO grades or primary and secondary glioblastomas, disregard concerning the infiltration zone or various epidemiological factors. The aim of our study was to evaluate MGMT expression and its prognostic value taking into consideration the aforementioned deficiencies. For this, 162 astrocytic tumors WHO II-IV (36 diffuse astrocytomas WHO II, 51 anaplastic astrocytomas, 75 primary glioblastomas) as well as 25 glioblastoma infiltration zones and 19 glioblastoma relapses were analyzed for immunohistochemical MGMT protein expression using tissue microarray technique. Expression of MGMT significantly decreased from WHO grade II $(25.6 \%)$ to glioblastoma $(16.8 \%, \mathrm{p}=0.01)$ with lowest levels in grade III tumors $(10.2 \%, \mathrm{II} / \mathrm{III} \mathrm{p}<0.0001)$. Significant negative associations of MGMT and survival were detected for WHO grade II and IV $(p=0.003$ and 0.013$)$. The optimal cut-off value of MGMT positive nuclei in primary glioblastomas discriminating patients with significantly different survival rates was at $15 \%$ $(\log -\operatorname{Rank} \mathrm{p}=0.0002)$. Individual relapse tumors showed changes of MGMT expression to a varying degree. The infiltration zone demonstrated a significant increase of MGMT $(p<0.0001)$. We conclude that immunohistochemical MGMT assessment has potential as a powerful diagnostic tool but analysis should only be performed in a grade dependent manner, before radio-/chemotherapy and with special attention to the infiltration zone of diffuse astrocytomas
\end{abstract}

DOI: https://doi.org/10.1007/s00401-007-0310-x

Posted at the Zurich Open Repository and Archive, University of Zurich ZORA URL: https://doi.org/10.5167/uzh-156400

Journal Article

Published Version

Originally published at:

Capper, David; Mittelbronn, Michel; Meyermann, Richard; Schittenhelm, Jens (2008). Pitfalls in the assessment of MGMT expression and in its correlation with survival in diffuse astrocytomas: proposal of a feasible immunohistochemical approach. Acta Neuropathologica, 115(2):249-259.

DOI: https://doi.org/10.1007/s00401-007-0310-x 


\title{
Pitfalls in the assessment of MGMT expression and in its correlation with survival in diffuse astrocytomas: proposal of a feasible immunohistochemical approach
}

\author{
David Capper · Michel Mittelbronn • \\ Richard Meyermann $\cdot$ Jens Schittenhelm
}

Received: 25 July 2007 / Revised: 5 October 2007 / Accepted: 6 October 2007 / Published online: 27 October 2007

(C) Springer-Verlag 2007

\begin{abstract}
Immunohistochemical studies showed that $\mathrm{O}_{6}$-methylguanine-DNA methyltransferase (MGMT) protein expression is negatively associated with survival in glioblastomas treated with alkylating agents in accordance with previous results of methylation-specific PCR. Implementation of this data in routine clinical diagnostics is limited due to often inappropriate study designs, e.g. pooling of tumor entities, WHO grades or primary and secondary glioblastomas, disregard concerning the infiltration zone or various epidemiological factors. The aim of our study was to evaluate MGMT expression and its prognostic value taking into consideration the aforementioned deficiencies. For this, 162 astrocytic tumors WHO II-IV (36 diffuse astrocytomas WHO II, 51 anaplastic astrocytomas, 75 primary glioblastomas) as well as 25 glioblastoma infiltration zones and 19 glioblastoma relapses were analyzed for immunohistochemical MGMT protein expression using tissue microarray technique. Expression of MGMT significantly decreased from WHO grade II $(25.6 \%)$ to glioblastoma $(16.8 \%, p=0.01)$ with lowest levels in grade III tumors $(10.2 \%$, II/III $p<0.0001)$. Significant negative associations of MGMT and survival were detected for WHO grade II and IV $(p=0.003$ and 0.013). The optimal cut-off value of MGMT positive nuclei in primary glioblastomas discriminating patients with significantly different survival rates was at $15 \%$ (Log-Rank $p=0.0002$ ). Individual relapse tumors showed changes of
\end{abstract}

D. Capper · M. Mittelbronn · R. Meyermann · J. Schittenhelm Institute of Brain Research, University of Tübingen,

Tübingen, Germany

D. Capper ( $\square)$

Institute of Neuropathology,

University of Zürich, Schmelzbergstrasse 12,

8091 Zürich, Switzerland

e-mail: David.Capper@usz.ch
MGMT expression to a varying degree. The infiltration zone demonstrated a significant increase of MGMT $(p<0.0001)$. We conclude that immunohistochemical MGMT assessment has potential as a powerful diagnostic tool but analysis should only be performed in a grade dependent manner, before radio-/chemotherapy and with special attention to the infiltration zone of diffuse astrocytomas.

Keywords MGMT $\cdot$ Astrocytoma ·

Immunohistochemistry

\section{Introduction}

Recently, the EORTC (European organisation for research and treatment of cancer) study with temozolomide in conjunction with radiotherapy after surgical resection demonstrated a significantly prolonged median patient survival, albeit only for single months [40]. Further analysis of the DNA-repair gene $M G M T\left(\mathrm{O}_{6}\right.$-methylguanine-DNA methyltransferase also known as AGT, NC-IUBMB: E.C.2.1.1.63) methylation status demonstrated a significant survival benefit solely for patients with a silenced MGMT gene as detected by methylation-specific polymerase-chainreaction analysis (MSP) in the group treated with radio-/ chemotherapy [16]. This confirmed previous results of a correlation of MGMT inactivation and clinical response to alkylating agents (e.g. temozolomide, nitrosourea) [11, 12, 18].

Besides MSP $[11,15,34,46]$ and RNA detecting methods [33, 42], the measurement of MGMT enzyme activity and the immunohistochemical (IHC) detection of MGMT protein have been employed repeatedly to assess the in vivo MGMT status [1, 8, 25, 39, 48]. In a recent comparison a significant correlation of IHC MGMT evaluation with MSP as well as MGMT enzyme activity was demonstrated, while 
discordance concerning the prognostic properties of MSP and IHC MGMT detection was assumed [23]. The explanatory power of this analysis remains limited though, as only a small number of pooled tumor samples were investigated and the prognostic groups were not confirmed with an actual survival benefit. If MGMT status is intended to guide future therapeutic practice the need for further standardization of MGMT testing is urgent.

Immunohistochemistry (IHC) presents several advantages for the detection of MGMT status, predominantly technical aspects (fast and easy to do, little material required, endothelia as internal positive control, material can be archived in paraffin, antibodies commercially available with mostly used clone mT3.1, see [5]) but also offers additional diagnostic information such as the visual discrimination of tumor cells from non-neoplastic cells. There is strong evidence for clonal heterogeneity of gliomas for MGMT methylation [2] and that modifications besides promoter methylation such as MGMT mutations or p53 derangement may affect the functional in vivo protein levels $[3,9]$. By measuring the endpoint of these regulations, IHC MGMT detection avoids several of these pitfalls. Nevertheless, IHC has several shortcomings, in particular tumor infiltration by MGMT positive inflammatory cells [29], as well as the possible induction of MGMT by radio-/chemotherapy $[13,47]$. The main problem with the interpretation of IHC MGMT status though, is an often insufficient study design. Glial tumors of various WHO grades with known prognostic differences or primary and secondary glioblastomas are pooled in a single group and analyzed for prognosis, the total sample size is often low, and cut-off values have been selected arbitrarily.

In the current study we have addressed several of the above shortcomings of IHC MGMT detection and attempted to establish non-arbitrary cut-off values for future prospective studies as well as routine clinical evaluation.

For this purpose, the IHC expression of MGMT is analyzed in a large array of diffuse astrocytomas of WHO grades II, III and IV, and associations with survival are evaluated independently for each grade. For grade IV, only primary glioblastomas at first occurrence are taken into analysis. To evaluate if radio-/chemotherapy has an impact on in vivo MGMT expression of glioblastomas, primary glioblastomas are investigated before and after therapy. Finally, special attention is given to infiltration versus central areas of glioblastomas.

\section{Materials and methods}

\section{Human tissue specimens}

A total of 162 brain tumor samples from the tumor bank of the Institute of Brain Research (Neuropathology), University of Tübingen were investigated. The patients underwent surgical treatment from 1993 to 2003 . The specimens consisted of $36 \mathrm{WHO}$ grade II astrocytomas (29 fibrillary, 5 gemistocytic, 2 protoplasmic; $19 \mathrm{M}, 17 \mathrm{~F}$, median age 38 years), 51 grade III anaplastic astrocytomas $(32 \mathrm{M}, 19 \mathrm{~F}$, median age 44 years) and 75 WHO grade IV glioblastomas ( $44 \mathrm{M}, 31 \mathrm{~F}$, median age 59 years), and they were diagnosed according to the WHO criteria for tumors of the nervous system by two senior neuropathologists (RM, MM) [20]. For grade IV, only primary glioblastomas were analyzed. Resection data as determined postoperatively by the neurosurgeon was available for 147 cases with 50 cases of complete macroscopic resection and 97 cases with incomplete resection. No tumor biopsies were integrated into this study. Additionally, 25 infiltration zones and 19 relapses of glioblastomas were included. All patients with relapse received field radiotherapy of up to 60 Gray and chemotherapy to a varying degree. Of six tumor specimens normal appearing white matter distant from the tumor was analyzed as a reference of MGMT expression in uninfiltrated brain. Usage of the tissue was approved by the local ethics committee.

Follow up for glioma patients was up to 12.6 years. Within this time frame a total of 99 patients died after a median survival of 13.9 months (range 1-151) whereas 54 were still alive with a median follow-up of 27.6 months (range 1-136). Nine patients were lost the trace immediately after operation. These patients were not included in survival analysis.

All specimens were fixed in $4 \%$ formalin $(\mathrm{pH} 7.4)$, embedded in paraffin and representative tissue samples were prepared as a tissue microarray (TMA) (punch diameter, $0.6 \mathrm{~mm}$ ) as previously described [27]. The TMA blocks were cut with a microtome ( $3 \mu \mathrm{m}$ thickness) and placed on SuperFrost Plus slides (Microm International, Walldorf, Germany).

Immunohistochemistry

The slides were deparaffinized in chloroform for $20 \mathrm{~min}$ then rehydrated in descending series of ethanol and washed in Tris-buffered saline (TBS). For antigen retrieval we used a simplified adaptation of the method introduced by Namimatsu and colleagues [30], boiling the slides in $0.05 \%$ citraconic anhydride ( $\mathrm{pH}$ 7.4) in a microwave oven at maximum power $(800 \mathrm{~W})$ for $20 \mathrm{~min}$, and then let the slides cool down to room temperature. Endogenous peroxidase was blocked with $3 \% \mathrm{H}_{2} \mathrm{O}_{2}$ in methanol for $15 \mathrm{~min}$, and standard swine serum was applied for $15 \mathrm{~min}$ to prevent nonspecific binding. As primary antibody we used monoclonal mouse anti-MGMT antibody clone MT3.1 (Chemicon, Temecula, USA) in a dilution of 1:75 and sections were incubated overnight at $4{ }^{\circ} \mathrm{C}$. Subsequent to 
washes in TBS for $10 \mathrm{~min}$, sections were incubated for 30 min with the secondary antibody at room temperature. The peroxidase-conjugated avidin-biotin complex (ABC) technique (Dako, Hamburg, Germany) with diaminobenzidine (DAB; Sigma, Deisenhofen, Germany) as chromogen was used to visualize the antigen binding. All sections were counterstained with hemalaun.

As negative controls (isotype control), every slide was also treated with replacement of primary antibody with a monoclonal $\mathrm{IgG}_{1}$ antibody for an Aspergillus niger surface protein (Dako Cytomation), a protein structure not expressed in mammalian tissue. None of the tumors showed an unspecific nuclear stain.

\section{Evaluation}

Evaluation of the immunohistochemical staining and photographic documentation was performed using an Olympus Vanox AHBT3 microscope. All slides were investigated individually by two experienced investigators and a mean score of both observations was used for statistics. Four cases were not possible to evaluate and were omitted from further analysis (see "Results"). At least 100 cells were evaluated for nuclear MGMT staining. Endothelial cells and perivascular lymphocytes were omitted from analysis. A weak staining of the nucleus was counted as positive. Granular staining of the nucleus was not observed. Cytoplasmic staining was not counted as positive.

\section{Statistical analysis}

The means were compared by a one-way analysis of variance (ANOVA) with subsequent Tukey-Kramer's HSD test with a global significance level of 5\%. For significant differences individual $p$ values were calculated. For individual pairwise comparisons we used a one sample $t$ test. For assessing correlations between age and MGMT expression an analysis of linear regression was used. The means are given together with their $95 \%$ confidence intervals (CI), significant differences are illustrated $(* p \leq 0.05$, $* * p \leq 0.01, * * * p \leq 0.0001)$. We chose a two-step survival analysis with the validation of a global association in an exponential parametric survival fit for each grade and a subsequent Kaplan Meier analysis. A significant global association with survival was set as a requirement to perform the dichotomy of the percental values for Kaplan Meier analysis. Grades II and IV met the above requirements and were repeatedly divided into two groups at 5\% intervals for optimal cut-off values. A designated group was never allowed to be less than five patients. For adjustment of the $p$ values due to multiple testing we used the method of Bonferroni. JMP IN (http://www.JMP.com) was used for statistical analysis.

\section{Results}

MGMT expression decreases significantly from low to high-grade gliomas

The vast majority of cases investigated demonstrated a moderate to strong nuclear stain for MGMT in at least some tumor cells (Fig. 1a-c). Generally, most endothelial nuclei as well as infiltrating inflammatory cells were strongly MGMT positive and endothelial nuclei served as an internal positive control. In endothelia, an additional weak cytoplasmic stain was regularly observed (Fig. 1a). As previously noted, we observed a droplet like cytoplasmic stain in few cases (one case of grade II, 11 cases of grade III) [24]. Matching these cases with the isotype control revealed that the staining does not represent cytoplasmic MGMT, but is nonspecific (Fig. 1d, e). Importantly, no cases with an unspecific nuclear reaction were observed in the isotype control. Four cases (one grade II, one grade III, two grade IV) could not be evaluated for MGMT expression by IHC, as the nuclear reaction was too weak to clearly differentiate positive and negative cells (Fig. 1f). The average levels of stained tumor cells were higher in low grade compared to high grade tumors with a mean expression of $25.6 \%$ (CI 20.1-31.1\%) in diffuse astrocytoma WHO grade II, decreasing to $10.2 \%$ (CI 5.6-14.8\%) in anaplastic astrocytoma grade III and $16.8 \%$ (CI 13.0-20.6\%) in glioblastoma WHO grade IV (Fig. 2). The decrease was significant (II/III $p<0.0001$; II/IV $p=0.01$ ) while the significance between grade III and IV was lost after correction for multiple testing (III/IV $p=0.031$ ). Six tumor cases with uninfiltrated white matter showed high levels of positive glial cells [mean 77.2\% (CI 60.7-93.6\%)] and all demonstrated a clear reduction of MGMT-positive nuclei in the tumor tissue (data not shown). Expression in uninfiltrated grey matter was lower than in white matter with neurons generally negative for MGMT (data not shown).

MGMT expression is significantly higher in glioblastoma infiltration zone compared to tumor center

A highly significant increase of nuclear MGMT expression was observed in the infiltration zone of the investigated glioblastomas compared to the corresponding tumor center $(p<0.0001)$. The mean expression was $43.9 \%$ (CI 35.3$52.5 \%$ ) in the infiltration zone compared to $16.1 \%$ (CI 7.7$24.5 \%$ ) in the subset of glioblastomas (Fig. 3). The mean expression of the infiltration zone was clearly lower than 

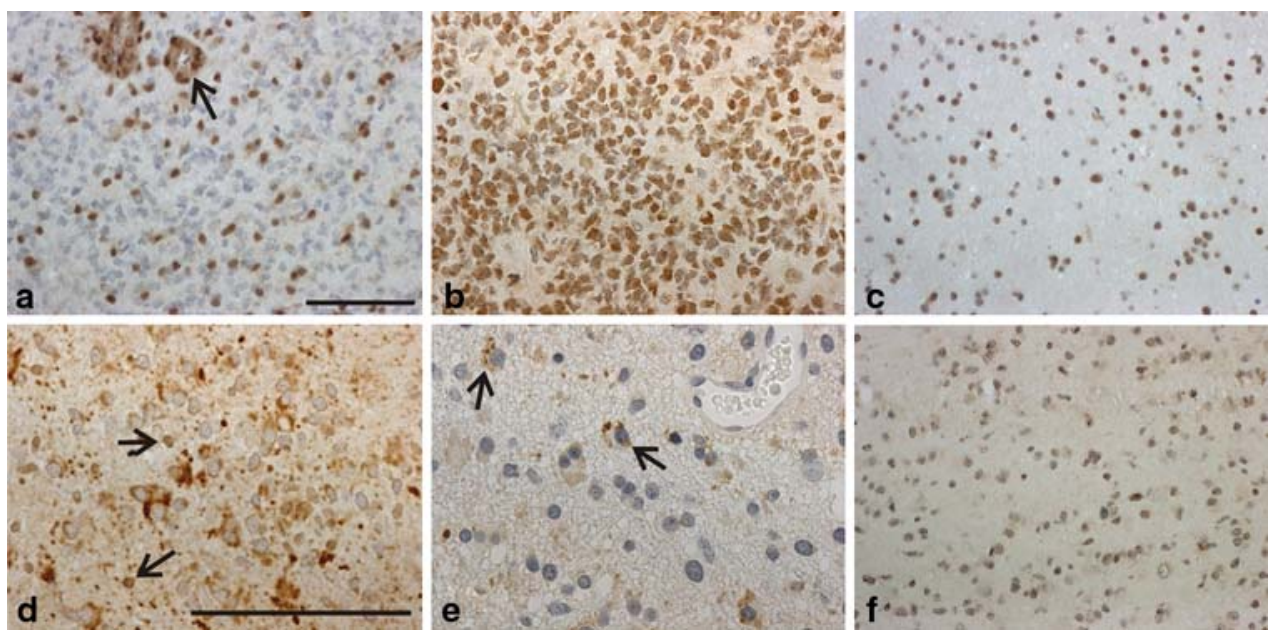

Fig. 1 Patterns of MGMT expression in diffuse astrocytomas. a Glioblastoma with disseminated MGMT positive cells while the majority of tumor cells are negative. Intermingled strongly positive endothelia with additional weaker cytoplasmic stain are also visible (arrow). b Different glioblastoma showing strong MGMT expression in majority of tumor cells. c Diffuse astrocytoma WHO II with strong MGMT expression in most cells. d Anaplastic astrocytoma with strong

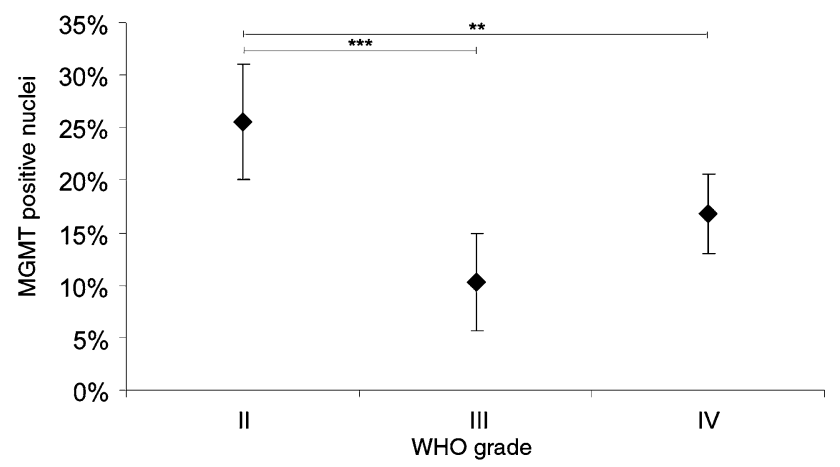

Fig. 2 Percental MGMT expression in astrocytoma WHO grade II-IV. Depiction of means and 95\% CI. A significant decrease was observed from WHO grade II to grade III and IV

the mean expression of uninfiltrated white matter (see above).

In vivo MGMT expression does not shift unidirectionally after radio-/chemotherapy

While the mean expression of MGMT in the group of relapse glioblastomas demonstrated an increase from $14.3 \%$ (CI 6.9-21.6\%) in the primary tumors to $20.4 \%$ (CI 13.1-27.7\%) in the relapses, a matched pairs analysis could not show significant differences $(p=0.20$; Fig. 4$)$. Several tumors demonstrated a considerable change of individual MGMT expression, but both, a clear increase as well as a droplet like cytoplasmic stain and single positive nuclei, most likely lymphocytes (arrows). e Although weaker, the cytoplasmic reaction is also visible in the isotype control of same tumor, delineating it as unspecific (arrows). f Diffuse astrocytoma WHO II with too weak MGMT expression to discriminate positive and negative cells. The tumor was omitted from further statistical analysis [magnification $\times 200$ (a-c and f); $\times 400$ (d and e); scale bar $100 \mu \mathrm{m}]$

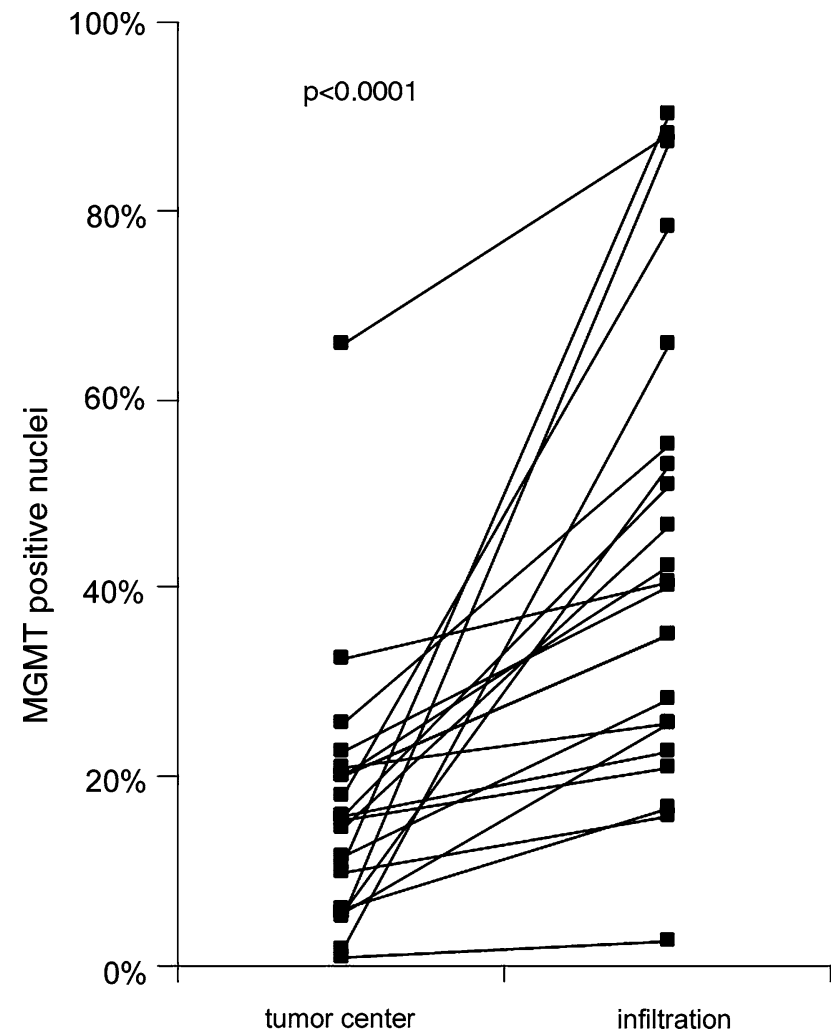

Fig. 3 Depiction of a matched pairs analysis of MGMT expression in tumor center and infiltration zone of glioblastoma. Corresponding pairs are connected by a line. All cases showed an increase in expression in the infiltration zone $(p<0.0001)$ 


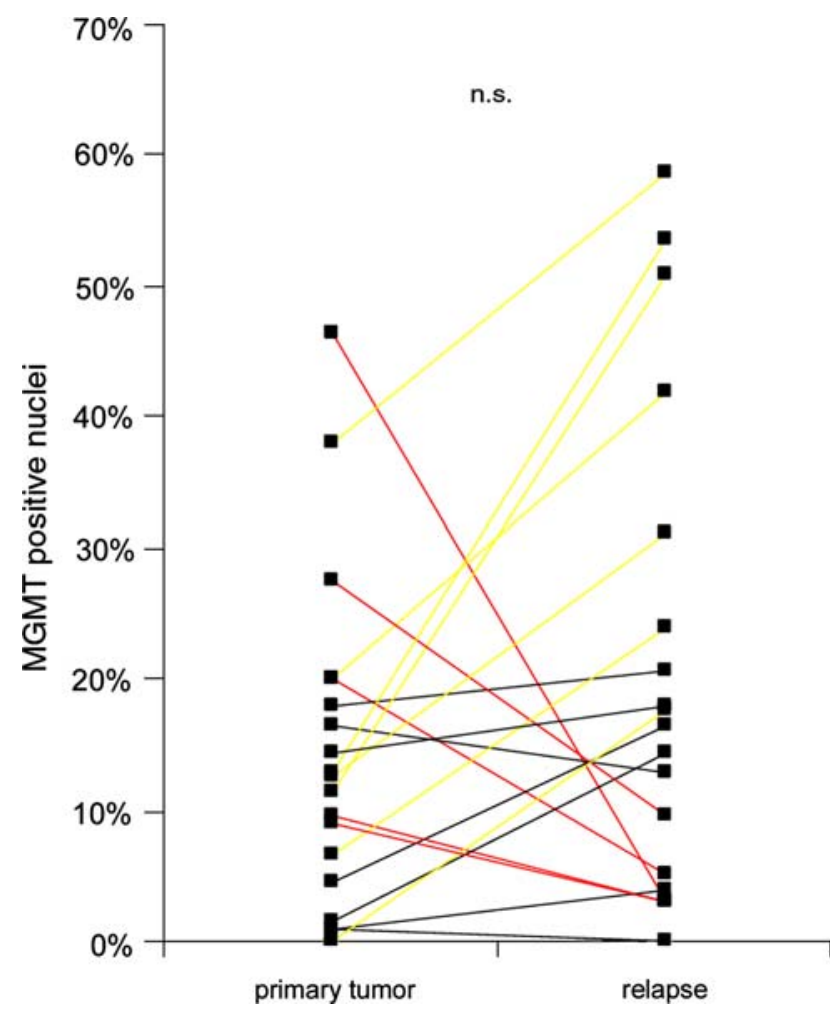

Fig. 4 Glioblastoma MGMT expression before and after therapy. Depiction of a matched pairs analysis of primary tumor and corresponding relapse (connected by line). Although the analysis did not indicate a significant unidirectional change of expression $(p=0.20)$, several individual tumors showed a marked alteration of nuclear MGMT. Red lines connect cases with a decrease beyond 95\% CI of mean difference, yellow lines an increase beyond $95 \%$ CI of mean difference, black lines are within $95 \% \mathrm{CI}$

clear decrease was observed. Three change of expression groups were distinguished in relation to the mean difference of all cases, one group with a clear decrease of MGMT (red, $n=5$ of 19) with a reduction beyond the CI of the

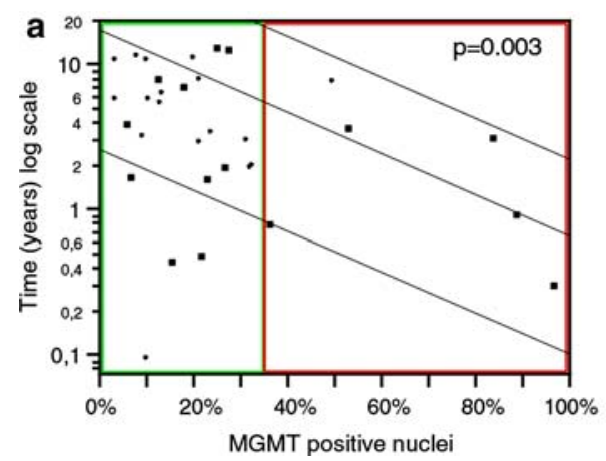

Fig. 5 MGMT expression is associated with survival in grade II astrocytoma with high levels correlating with a worse survival. a Depiction of exponential parametric survival fit (note the log scale) with survival time in relation to MGMT expression (\%). Squares reached final end point, small dots represent censored data. The analysis demonstrates a highly significant association of worse survival with high expression mean difference, the second with no individual changes (black, $n=7$ of 19) (difference within 95\% CI) and the third with a clear increase of MGMT out of the range of the $95 \%$ CI (yellow, $n=7$ of 19). No further association of the groups with individual chemotherapy was seen (data not shown).

MGMT is negatively associated with survival in WHO grade II and IV

The survival analysis for WHO grade II, III and IV tumors showed a significant association in the parametric exponential survival fit of grades II and IV $(p=0.003$ and 0.013 , respectively; Figs. 5, 6) while grade III tumors demonstrated no such association $(p=0.18$, data not shown). Even for the subgroup of WHO grade III tumors that received an alkylating therapy $(n=19)$ no significant results were obtained ( $p=0.88$, data not shown). For both grades II and IV, low levels of MGMT were associated with a better patient survival. Further analysis for an optimal cut-off value for prognostic evaluation demonstrated that for grade II, MGMT expression in 35\% of the tumor cells was the delimiter with the best $p$ value (Table 1). The median survival was 44.4 months (mean 63.8, range 1-151) for low MGMT expression in contrast to 10.4 months (mean 31.6, range 3-89) in the high MGMT group. The division of WHO grade II cases at $35 \%$ led to two groups of clearly different size, indicating that a small group of patients with high MGMT expression $(n=6)$ were predominantly responsible for the association with survival.

In grade IV the optimal value for a dichotomy was found at $15 \%$ MGMT positive cells (Table 1). This value highly discriminated between patients with a good prognosis and patients with a poor prognosis (Log-Rank $p=0.0002$; Fig. 6). Median survival for the group with low expression

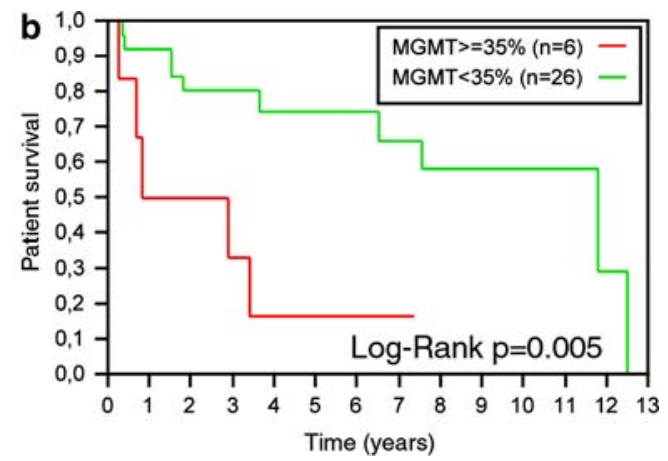

$(p=0.003)$. For illustration, the groups for Kaplan Meier analysis are surrounded by a green and a red box, splitting the cases at 35\% MGMT expression. b Kaplan Meier analysis of same data demonstration a highly significant discrimination of the two prognostic groups (LogRank $p=0.005$ ) 
Fig. 6 MGMT expression is associated with survival in glioblastoma with high levels correlating with a worse survival (as in Fig. 5, except for splitting the boxes at a cut-off value of $15 \%$ )
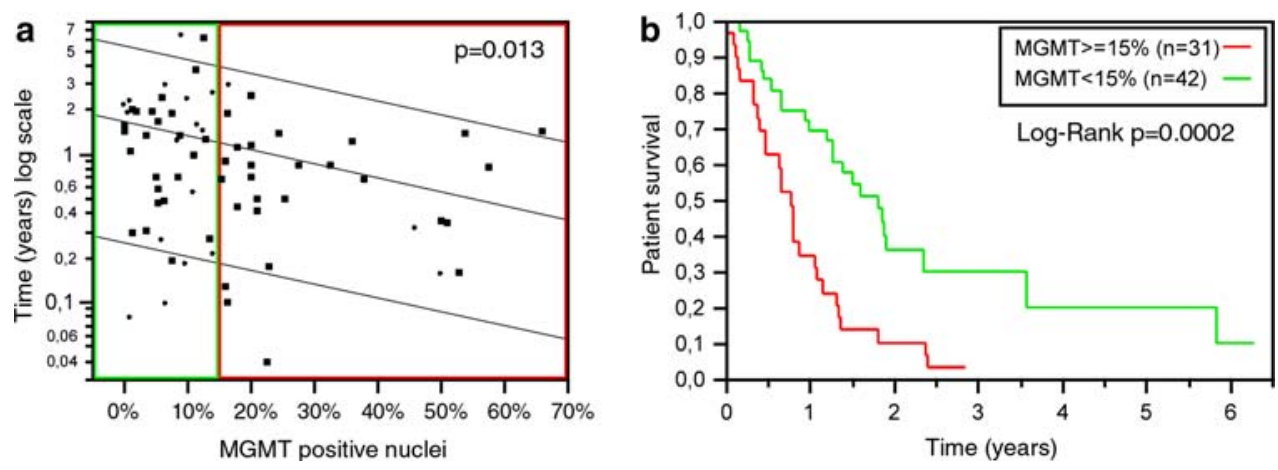

Table 1 Log-Rank $p$-values of Kaplan Meier analysis for the cut-off at different percental MGMT expression for WHO grade II and IV

\begin{tabular}{lll}
\hline Cut-off level (\%) & WHO II & WHO IV \\
\hline 5 & n.a. & $p=0.42$ \\
10 & $p=0.68$ & $p=0.13$ \\
15 & $p=0.12$ & $p=0.0002$ \\
20 & $p=0.3$ & $p=0.001$ \\
25 & $p=0.29$ & $p=0.004$ \\
30 & $p=0.08$ & $p=0.02$ \\
35 & $p=0.005$ & $p=0.02$ \\
40 & n.a. & $p=0.04$ \\
50 & $p=0.03$ & $p=0.03$ \\
\hline
\end{tabular}

The $p$ value for a significant result after Bonferroni correction was $p<0.007$ for grade II and $p<0.006$ for grade IV

n.a. Not applicable

was 15.4 months (mean 17.9, range 1-75) compared to 8.0 months (mean 10.2, range 1-34) for the group of high MGMT expression. The differences in group-size at this cut-off value were within reasonable limits.

\section{Correlation of MGMT expression and patient age}

In the analysis of associations between MGMT expression and patient age a marginally significant positive correlation was detected for grade II and a strong trend for grade IV ( $p=0.046$ and 0.052 ; Fig. 7), whereas WHO grade III displayed no association ( $p=0.27$; data not shown).

As for grade II all patients with a worse prognosis were old (Fig. 7a), it seems evident that for grade II the association of MGMT expression and survival was predominantly by the difference of age, which is a well-known predictive factor of survival.

MGMT retains its association with survival in peak age group of glioblastoma

To verify that the association of MGMT with survival in glioblastoma is not an effect of its near correlation with age (Fig. 7b), glioblastoma cases were reduced to the peak age group of patients from 45 to 70 years [20], thus omitting patients with extreme age deviations from the analysis. Even for the smaller sample group the significance for the association with survival remained in the exponential parametric survival fit ( $p=0.035$; Fig. 8a) as well as in the Kaplan Meier analysis with a significant discrimination of patients with good and bad prognosis at the previously established cut-off value of $15 \%$ ( $p=0.011$; Fig. 8b). For the reduced group, no association of patient age and MGMT expression was detectable $(p=0.147$, data not shown).

\section{Discussion}

Future glioma therapy will consider tumor MGMT status and possibly stratify patients by their MGMT expression [14]. Several studies using methods such as MSP and IHC have demonstrated an association of MGMT status and survival (IHC [1, 7, 12, 29, 35]; MSP [10, 11, 15, 16, 34, 45]) while other groups could not or only partially detect associations with survival $[2,4,19]$. Recently in reaction to an IHC study of MGMT in inoperable newly diagnosed glioblastoma patients [7], MSP was clearly advocated for the detection of MGMT status [41].

It is worth mentioning that MSP also has distinct shortcomings. Astrocytomas are very heterogeneous tumors, both in histology and (epi)-genetics, and this is also true for the methylation of the $M G M T$ promoter as demonstrated by Blanc et al. [2]. In a recent comparison of IHC and MSP, Maxwell noted that even in MSP negative tumors (where $M G M T$ is not silenced) a large fraction of potentially temozolomide sensitive MGMT negative cells is present and in MSP positive tumors (where MGMT is silenced) large fractions of MGMT positive cells can be found. MSP has the great advantage of offering an explicit MGMT status, though it seems as if the method artificially divides gliomas into black and white, and does not take into account the heterogeneous nature of these tumors. This makes therapeutic decisions on the basis of MSP status alone somewhat unsatisfactory. It is possible, that $M G M T$ methylation status 


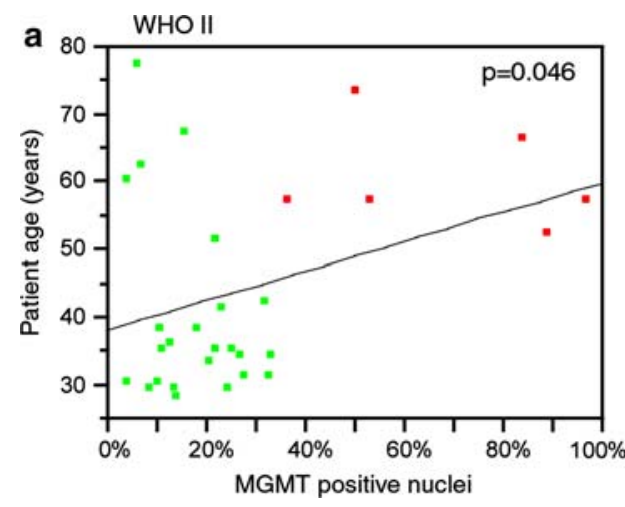

Fig. 7 Correlation of age and MGMT in grade II and IV. Green squares correspond to good prognosis group, red to bad prognosis (see Figs. 5 and 6). a The depiction of grade II MGMT expression in relation to age shows that all patients with a high expression were older than 50 years. No patients under 50 years were in the group of bad

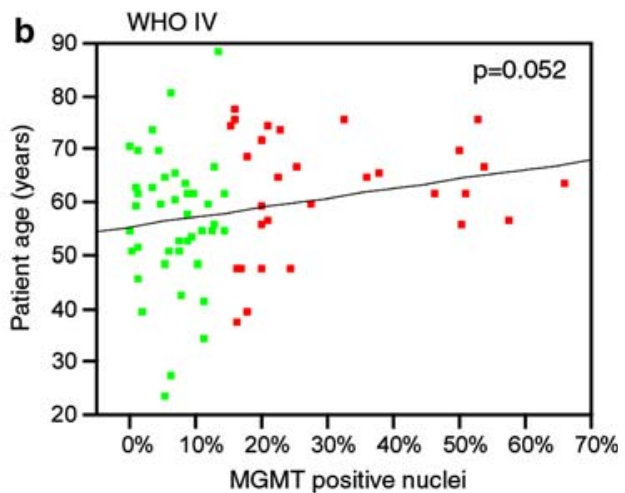

prognosis. The linear regression reached marginal significance $(p=0.046)$. b For grade IV the association showed a strong trend $(p=0.052)$ but did not reach significance. Likewise, the illustration shows that higher expression (above $25 \%$ ) is exclusively found in older patients

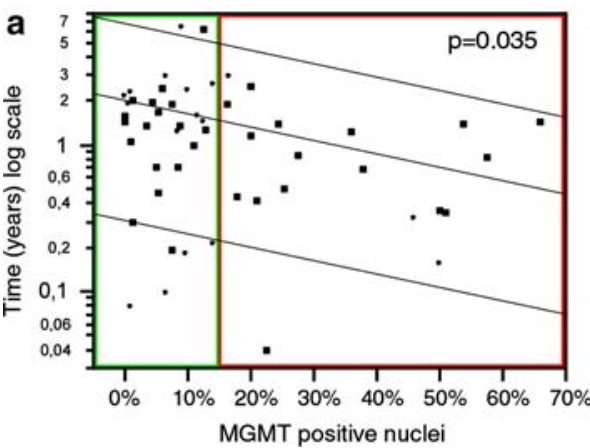

Fig. 8 Even in the reduced peak age group of glioblastoma MGMT retains its association with survival. a Illustration of exponential parametric survival fit demonstrating a significant association of survival

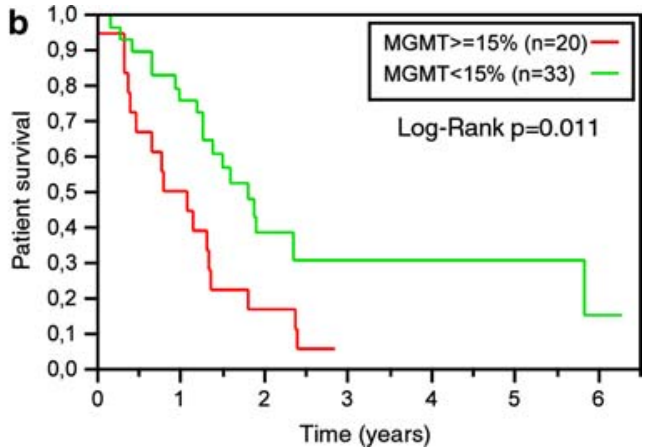

and MGMT even in the peak age group of glioblastoma (45-70 years). b Kaplan Meier analysis at the previously established cut-off value of $15 \%$ also remains significant for the peak age group

to high-grade diffuse astrocytoma, an observation that is supported by other studies [26, 36, 48] and clear differences between grade III and IV tumors. This clearly disqualifies the pooling of astrocytoma grades for survival analysis. Our study did not integrate secondary glioblastomas for the reasons that (1) the genetic pathways leading to primary or secondary glioblastoma differ at several levels [32], (2) secondary glioblastomas only constitute a small subgroup of glioblastomas [31], (3) p53 levels, known to influence MGMT status, clearly differ in primary and secondary glioblastoma [44], and (4) the methylation of MGMT promoter shows significantly higher levels in secondary glioblastomas [28].

All these factors may in part explain the wide range of cut-offs in use for "high-grade" glioma ranging from $<5$ to $35 \%[1,4,7,12,29,35]$. As Table 2 indicates, most IHC studies conducted so far pooled several WHO grades, some even oligodendroglial and astrocytic tumors. Of the two studies with values exclusive for grade IV, Chinot et al. [7] investigated the subgroup of inoperable tumors, a group were able to demonstrate a significant decrease from low 
Table 2 IHC studies determining a cut-off value for MGMT methylation status

\begin{tabular}{|c|c|c|c|c|c|c|}
\hline Study & $\begin{array}{l}\text { MGMT } \\
\text { antibody } \\
\text { clone }\end{array}$ & $\begin{array}{l}\text { Cut-off for } \\
\text { prognostic } \\
\text { groups (\%) }\end{array}$ & $\begin{array}{l}\text { Number of } \\
\text { cases and WHO } \\
\text { grade }\end{array}$ & $\begin{array}{l}\text { Association } \\
\text { with overall } \\
\text { survival }\end{array}$ & Special & $\begin{array}{l}\text { Primary/secondary } \\
\text { glioblastoma }\end{array}$ \\
\hline Friedman et al. [12] & MT3.1 & 20 & 38; 5 AA (III), 33 IV & n.a. & 37\% Biopsy only & Primary \\
\hline Anda et al. [1] & $\begin{array}{l}\text { Mouse } \\
\text { monoclonal }\end{array}$ & 19 & $18 ; \mathrm{IV}$ & $p=0.01$ & & Primary \\
\hline Nakasu et al. [29] & MT3.1 & 10 & $61 ;$ III and IV & $p=0.009$ & & Not declared \\
\hline Brell et al. [4] & MT3.1 & $<5$ & $\begin{array}{l}93 ; 75 \text { AA (III), } \\
18 \text { AO\&AOA (III) }\end{array}$ & $p=0.066$ & $33 \%$ Biopsy only & $\begin{array}{l}\text { Newly diagnosed } \\
\text { WHO grade III }\end{array}$ \\
\hline Pollack et al. [35] & $\mathrm{mT} 23.2$ & $>25$ & 109; III and IV & $p=0.17$ & Childhood glioblastoma & Primary \\
\hline Chinot et al. [7] & MT3.1 & 35 & 25; IV & $p=0.004$ & Inoperable tumors only & Primary \\
\hline
\end{tabular}

$A A$ Anaplastic astrocytoma, $A O$ anaplastic oligodendroglioma, $A O A$ anaplastic oligoastrocytoma, $n . a$. not applicable

with a survival not comparable to the general patient collective and in the study of Anda et al. [1] analysis was limited to 18 primary glioblastomas. Further factors that are likely to influence the results are technical factors of the IHC procedure and interobserver variability. For a standardized IHC procedure we performed an adoption of the antigen retrieval method of Namimatsu et al. [30]. Interobserver variability should be evaluated in a larger multi-institutional study to determine the number of patients ending up in divergent prognostic groups, thereby further elucidating the feasibility of IHC MGMT status detection with our proposed cut-off.

In this study on astrocytic tumors, only grade II and IV tumors demonstrated an association with survival, whereas grade III tumors did not. Likewise, Brell demonstrated a lack of association of MGMT methylation with survival as well as MGMT IHC for a pooled group of grade III gliomas (anaplastic astrocytoma, anaplastic oligodendroglioma, and anaplastic oligoastrocytoma) [4]. It is noteworthy that Brell and colleagues demonstrated an association of IHC and survival in a secondary analysis of a subgroup of patients treated with an alkylating agent, whereas the same analysis remained insignificant in our cohort. Thus, it is possible that the results of Brell were strongly influenced by the inclusion of tumors with an oligodendroglial differentiation which are potentially more susceptible to chemotherapy [6], whereas for anaplastic astrocytomas even post-alkylating therapy survival seems to be independent of MGMT status. Our results give new input to the current discussion on the lack of justification for temozolomide as the first line treatment for anaplastic astrocytoma (reviewed in [38]). In contrast, MGMT expression was elevated in WHO grade II tumors and uninfiltrated white matter, indicating that their DNA repair mechanisms function on a higher level. In general, this seems desirable, as high MGMT levels are likely to counteract genetic alteration (e.g. spontaneous mutations by endogenous DNA alkylation) that might lead to tumor development and progression [22]. For the treatment of low-grade astrocytomas this poses a problem though, as several therapeutic substances depend on a reduced DNA repair mechanism of tumor cells.

A recent MGMT methylation study of 27 fibrillary astrocytomas grade II could not detect a significant relationship with overall survival [46] while a cohort of 49 grade II astrocytomas identified MGMT methylation even as an adverse indicator of recurrence free survival [21]. Data concerning the time to relapse could not be integrated into this study because of the small number of assessable cases. Two explanations for the discrepancy to our results are conceivable. (1) Our group of grade II tumors did not entirely consist of fibrillary astrocytomas given that five gemistocytic and two protoplasmic tumors were also included. For gemistocytic astrocytomas a more aggressive behavior has been described [20]. In an analysis omitting these tumors, the parametric survival fit remained significant $(p=0.016)$, proving that these tumors are not causing the discrepancy. (2) Our data for grade II demonstrated a significant positive correlation to patient age with expression above $35 \%$ exclusively found in patients above 50 years. To our knowledge, a significant positive association of age and MGMT expression in WHO grade II tumors has not been described to date. This association may partially contribute to the very strong prognostic power of age in grade II tumors [31]; on the other hand it complicates the interpretation of the presented data. Our cut-off of $35 \%$ for grade II, forming one small group of old patients with high expression and bad prognosis does not suit the standards that we aimed to establish by this study and therefore we suggest further evaluation on the significance of MGMT in diffuse astrocytoma WHO grade II.

Our study represents the largest observation of IHC MGMT expression in primary glioblastoma before treatment and offers a highly significant cut-off value for the further evaluation of prognostic groups. To our knowledge a 
trend of MGMT increase with age in glioblastoma has not been described yet. In contrast, Silber et al. demonstrated that MGMT enzyme activity is inversely correlated with age in glioblastoma [39] and results of semi-quantitative RT-PCR indicate an equivalent correlation on the gene expression level in primary glioblastomas [37]. The discrepancy of high protein levels and low enzyme activity could possibly be explained by several loss of function mutations in the MGMT gene that have been identified in mutagenesis studies, although the in vivo prevalence of these mutations has not been documented [9]. However, the results of other studies could demonstrate a significant correlation of MGMT enzyme activity and immunohistochemical labeling [23], casting the above explanations into doubt and offering a sampling effect as a more likely reason for the discrepancy.

A further aspect we tried to elucidate with this study was the proposed induction of MGMT by radio-/chemotherapy $[13,47]$. For this, a cohort of primary glioblastomas was analyzed before and after radiotherapy and alkylating chemotherapy. No statistically significant unidirectional change of MGMT expression was detected although almost three-quarter of the tumors showed marked changes out of the CI range of their MGMT profile. The cause of these changes remains enigmatic. In contrast, Nakasu et al. [29] could not detect changes of MGMT expression in recurrent gliomas in a smaller cohort. Still, our results lead us to the conclusion that considerable changes can be observed after therapy and our proposed cut-off value of $15 \%$ should only be applied before application of radio-/chemotherapy. In case of a relapse the reevaluation of MGMT tumor status seems necessary.

Besides chemotherapy other drugs have also been implicated in interfering with MGMT expression with contrary results found in literature, foremost glucocorticoids [17, 43]. Although many of our high-grade astrocytoma patients received dexamethasone, the dosages and application intervals before operation were not traceable and no statistical analysis regarding this question could be performed.

In an analysis of further possible sources of error the infiltration zone of diffuse astrocytoma demonstrated a considerable increase of MGMT. It is important to note that a clear histological discrimination of tumor cells and infiltrated normal brain cells was not possible. As we observed high levels of MGMT in normal brain astrocytes and oligodendrocytes, the high levels in the infiltration zone most probably represents residual non-neoplastic cells [29]. This finding is problematic for several methods of MGMT detection. In IHC, small samples of tissue might only contain infiltration zone and might therefore be perceived as falsely high MGMT expressing tumors; MSP analysis might reveal false negative results as the proportion of tumor cells in the selected material might be too low. Highgrade gliomas are further known to be infiltrated by MGMT positive non-neoplastic cells (mostly lymphocytes and macrophages/microglia) constituting up to $7 \%$ of the total tumor cells [29]. In IHC, it is often not possible to distinguish monocytes from neoplastic astrocytes in routine staining and routine immunohistochemical double staining is not practicable. As our proposed cut-off of $15 \%$ lies well beyond the observed maximum of $7 \%$ non-neoplastic cells, we conclude that these cells are generally summed up in the group of low expression and in general will not further interfere with the evaluation.

As we aspire to propose a standardized procedure for immunohistochemical evaluation several points for routine diagnostics need to be addressed: What region of the tumor should be evaluated? In this study we selected areas with the most prominent signs of atypia/anaplasia, especially cellular density and nuclear polymorphism. Still cases with diverging MGMT expression in equally atypical regions may be observed; in this case we propose evaluating the area with the higher staining indices as these cells supposedly determine survival. When is an MGMT stained cell counted as positive? Equivalent to the staining of p53 and other nuclear markers like progesterone receptor the MGMT staining is not of an on/off type, as every shade of staining was detected. This is a risk for a significant observer bias. In our investigation we decided to even count weak staining as positive, as results with the highly sensitive nested in situ RT-PCR indicated that immunohistochemical evaluation underrate the actual MGMT protein expression [33]. What about the infiltration zone? We propose that immunohistochemical evaluation should only be performed when the tumor can be unequivocally identified. Especially in low-grade diffuse astrocytoma this can cause serious problems. In cases of uncertainty we propose not to evaluate MGMT by IHC alone. What cells should be evaluated? We propose to count all cells except vascular endothelia and perivascular lymphocytes as our cut-off is sufficiently high to ensure that counted macrophages/ microglia will not alter prognostic status. A further discrimination is simply not workable for fast routine diagnostics.

In conclusion, we were able to demonstrate that the evaluation of MGMT status for glioma should be performed strictly grade dependent. We propose further investigations of the relation of survival and MGMT expression in grade II and urge to analyze the effect of age adequately in further studies. The lack of association with survival in grade III may be a representation of the different biological behavior and altered response to chemotherapy although further studies are needed to verify our results. As even the patients receiving alkylating therapeutics showed no association of survival and MGMT, possibly other first-line therapeutic approaches should be sought for WHO grade III astrocytomas. 
For de-novo grade IV glioblastomas we were able to establish an optimal cut-off value of $15 \%$ MGMT positive nuclei for maximal discrimination of prognostic groups.

Finally, we conclude that the current methods of identifying MGMT status should not lead to a status dependent therapy with possibly not treating MGMT positive patients, as the heterogeneous aspect of every investigated tumor indicates a fraction of potentially chemotherapy sensitive cells. If patient tailored therapy is sought, further understanding of MGMT regulation and further molecular determinants of survival are urgently needed.

Acknowledgments We thank R. Ajaaj, G. Tabatabai and F. Stubenvoll for the collection of the clinical data and B. J. Capper for critical reading of the manuscript.

\section{References}

1. Anda T, Shabani HK, Tsunoda K, Tokunaga Y, Kaminogo M, Shibata S, Hayashi T, Iseki M (2003) Relationship between expression of O6-methylguanine-DNA methyltransferase, glutathione-S-transferase pi in glioblastoma and the survival of the patients treated with nimustine hydrochloride: an immunohistochemical analysis. Neurol Res 25:241-248

2. Blanc JL, Wager M, Guilhot J, Kusy S, Bataille B, Chantereau T, Lapierre F, Larsen CJ, Karayan-Tapon L (2004) Correlation of clinical features and methylation status of MGMT gene promoter in glioblastomas. J Neurooncol 68:275-283

3. Blough MD, Zlatescu MC, Cairncross JG (2007) O6-methylguanine-DNA methyltransferase regulation by $\mathrm{p} 53$ in astrocytic cells. Cancer Res 67:580-584

4. Brell M, Tortosa A, Verger E, Gil JM, Viñolas N, Villá S, Acebes JJ, Caral L, Pujol T, Ferrer I, Ribalta T, Graus F (2005) Prognostic significance of O6-methylguanine-DNA methyltransferase determined by promoter hypermethylation and immunohistochemical expression in anaplastic gliomas. Clin Cancer Res 11:5167-5174

5. Brent TP, von Wronski M, Pegram CN, Bigner DD (1990) Immunoaffinity purification of human O6-alkylguanine-DNA alkyltransferase using newly developed monoclonal antibodies. Cancer Res 50:58-61

6. Cairncross G, Berkey B, Shaw E, Jenkins R, Scheithauer B, Brachman D, Buckner J, Fink K, Souhami L, Laperierre N, Mehta M, Curran W (2006) Phase III trial of chemotherapy plus radiotherapy compared with radiotherapy alone for pure and mixed anaplastic oligodendroglioma: intergroup radiation therapy oncology group trial 9402. J Clin Oncol 24:2707-2714

7. Chinot OL, Barrié M, Fuentes S, Eudes N, Lancelot S, Metellus P, Muracciole X, Braguer D, Ouafik L, Martin PM, Dufour H, Figarella-Branger D (2007) Correlation between O6-methylguanine-DNA methyltransferase and survival in inoperable newly diagnosed glioblastoma patients treated with neoadjuvant temozolomide. J Clin Oncol 25:1470-1475

8. Citron M, White A, Decker R, Wasserman P, Li B, Randall T, Guerra D, Belanich M, Yarosh D (1996) O6-methylguanine-DNA methyltransferase in human brain tumors detected by activity assay and monoclonal antibodies. Oncol Res 7:49-55

9. Crone TM, Goodtzova K, Pegg AE (1996) Amino acid residues affecting the activity and stability of human O6-alkylguanineDNA alkyltransferase. Mutat Res 363:15-25

10. Donson AM, Addo-Yobo SO, Handler MH, Gore L, Foreman NK (2007) MGMT promoter methylation correlates with survival benefit and sensitivity to temozolomide in pediatric glioblastoma. Pediatr Blood Cancer 48:403-407

11. Esteller M, Garcia-Foncillas J, Andion E, Goodman SN, Hidalgo OF, Vanaclocha V, Baylin SB, Herman JG (2000) Inactivation of the DNA-repair gene MGMT and the clinical response of gliomas to alkylating agents. N Engl J Med 343:1350-1354

12. Friedman HS, McLendon RE, Kerby T, Dugan M, Bigner SH, Henry AJ, Ashley DM, Krischer J, Lovell S, Rasheed K, Marchev F, Seman AJ, Cokgor I, Rich J, Stewart E, Colvin OM, Provenzale JM, Bigner DD, Haglund MM, Friedman AH, Modrich PL (1998) DNA mismatch repair and O6-alkylguanine-DNA alkyltransferase analysis and response to Temodal in newly diagnosed malignant glioma. J Clin Oncol 16:3851-3857

13. Fritz G, Tano K, Mitra S, Kaina B (1991) Inducibility of the DNA repair gene encoding O6-methylguanine-DNA methyltransferase in mammalian cells by DNA-damaging treatments. Mol Cell Biol 11:4660-4668

14. Hau P, Stupp R, Hegi ME (2007) MGMT methylation status: the advent of stratified therapy in glioblastoma? Dis Markers 23:97104

15. Hegi ME, Diserens AC, Godard S, Dietrich PY, Regli L, Ostermann S, Otten P, Van Melle G, de Tribolet N, Stupp R (2004) Clinical trial substantiates the predictive value of O-6-methylguanine-DNA methyltransferase promoter methylation in glioblastoma patients treated with temozolomide. Clin Cancer Res 10:1871-1874

16. Hegi ME, Diserens AC, Gorlia T, Hamou MF, de Tribolet N, Weller M, Kros JM, Hainfellner JA, Mason W, Mariani L, Bromberg JE, Hau P, Mirimanoff RO, Cairncross JG, Janzer RC, Stupp R (2005) MGMT gene silencing and benefit from temozolomide in glioblastoma. N Engl J Med 352:997-1003

17. Hermisson M, Klumpp A, Wick W, Wischhusen J, Nagel G, Roos W, Kaina B, Weller M (2006) O6-methylguanine DNA methyltransferase and p53 status predict temozolomide sensitivity in human malignant glioma cells. J Neurochem 96:766-776

18. Jaeckle KA, Eyre HJ, Townsend JJ, Schulman S, Knudson HM, Belanich M, Yarosh DB, Bearman SI, Giroux DJ, Schold SC (1998) Correlation of tumor O6 methylguanine-DNA methyltransferase levels with survival of malignant astrocytoma patients treated with bis-chloroethylnitrosourea: a southwest oncology group study. J Clin Oncol 16:3310-3315

19. Kamiryo T, Tada K, Shiraishi S, Shinojima N, Kochi M, Ushio Y (2004) Correlation between promoter hypermethylation of the O6methylguanine-deoxyribonucleic acid methyltransferase gene and prognosis in patients with high-grade astrocytic tumors treated with surgery, radiotherapy, and 1-(4-amino-2-methyl-5-pyrimidinyl)methyl-3-(2-chloroethyl)-3-nitrosourea-based chemotherapy. Neurosurgery 54:349-357

20. Kleihues P, Cavenee WK (2000) Pathology and genetics of tumours of the nervous system. IARC Press, Lyon

21. Komine C, Watanabe T, Katayama Y, Yoshino A, Yokoyama T, Fukushima T (2003) Promoter hypermethylation of the DNA repair gene O6-methylguanine-DNA methyltransferase is an independent predictor of shortened progression free survival in patients with low-grade diffuse astrocytomas. Brain Pathol 13:176-184

22. Margison GP, Povey AC, Kaina B, Santibáñez Koref MF (2003) Variability and regulation of O6-alkylguanine-DNA alkyltransferase. Carcinogenesis 24:625-635

23. Maxwell JA, Johnson SP, Quinn JA, McLendon RE, Ali-Osman F, Friedman AH, Herndon JE, Bierau K, Bigley J, Bigner DD, Friedman HS (2006) Quantitative analysis of O6-alkylguanineDNA alkyltransferase in malignant glioma. Mol Cancer Ther 5:2531-2539

24. McLendon RE, Cleveland L, Pegram C, Bigner SH, Bigner DD, Friedman HS (1998) Immunohistochemical detection of the DNA 
repair enzyme O6-methylguanine-DNA methyltransferase in formalin-fixed, paraffin-embedded astrocytomas. Lab Invest 78:643-644

25. Mineura K, Izumi I, Watanabe K, Kowada M (1991) O6-alkylguanine-DNA alkyltransferase activity in human brain tumors. Tohoku J Exp Med 165:223-228

26. Mineura K, Yanagisawa T, Watanabe K, Kowada M, Yasui N (1996) Human brain tumor O(6)-methylguanine-DNA methyltransferase mRNA and its significance as an indicator of selective chloroethylnitrosourea chemotherapy. Int J Cancer 69:420-425

27. Mittelbronn M, Capper D, Bunz B, Dietz K, Goeppert B, Ajaaj R, Tabatabai G, Stubenvoll F, Schlaszus H, Merseburger AS, Becker R, Freudenstein D, Wick W, Weller M, Meyermann R, Simon P (2007) De novo erythropoietin receptor (EPO-R) expression in human neoplastic glial cells decreases with grade of malignancy but is favourably associated with patient survival. Neuropathol Appl Neurobiol 33:299-307

28. Nakamura M, Watanabe T, Yonekawa Y, Kleihues P, Ohgaki H (2001) Promoter methylation of the DNA repair gene MGMT in astrocytomas is frequently associated with $\mathrm{G}: \mathrm{C} \rightarrow \mathrm{A}: \mathrm{T}$ mutations of the TP53 tumor suppressor gene. Carcinogenesis 22:1715-1719

29. Nakasu S, Fukami T, Baba K, Matsuda M (2004) Immunohistochemical study for O6-methylguanine-DNA methyltransferase in the non-neoplastic and neoplastic components of gliomas. J Neurooncol 70:333-340

30. Namimatsu S, Ghazizadeh M, Sugisaki Y (2005) Reversing the effects of formalin fixation with citraconic anhydride and heat: a universal antigen retrieval method. J Histochem Cytochem 53:3-11

31. Ohgaki H, Kleihues P (2005) Population-based studies on incidence, survival rates, and genetic alterations in astrocytic and oligodendroglial gliomas. J Neuropathol Exp Neurol 64:479-489

32. Ohgaki H, Kleihues $P$ (2007) Genetic pathways to primary and secondary glioblastoma. Am J Pathol 170:1445-1453

33. Ohe N, Saio M, Kijima M, Tamakawa N, Suwa T, Kojima Y, Yano H, Kaku Y, Iwama T, Shinoda J, Sakai N, Takami T (2003) In situ detection of O6-methylguanine-DNA methyltransferase messenger RNA in paraffin-embedded human astrocytic tumor tissues by nested in situ RT-PCR is useful in predicting chemotherapy-resistance of tumors. Int J Oncol 22:543-549

34. Paz MF, Yaya-Tur R, Rojas-Marcos I, Reynes G, Pollan M, Aguirre-Cruz L, García-Lopez JL, Piquer J, Safont MJ, Balaña C, Sanchez-Cespedes M, García-Villanueva M, Arribas L, Esteller M (2004) CpG island hypermethylation of the DNA repair enzyme methyltransferase predicts response to temozolomide in primary gliomas. Clin Cancer Res 10:4933-4938

35. Pollack IF, Hamilton RL, Sobol RW, Burnham J, Yates AJ, Holmes EJ, Zhou T, Finlay JL (2006) O6-methylguanine-DNA methyltransferase expression strongly correlates with outcome in childhood malignant gliomas: results from the CCG-945 Cohort. J Clin Oncol 24:3431-3437

36. Preuss I, Haas S, Eichhorn U, Eberhagen I, Kaufmann M, Beck T, Eibl RH, Dall P, Bauknecht T, Hengstler J, Wittig BM, Dippold W, Kaina B (1996) Activity of the DNA repair protein O6-
methylguanine-DNA methyltransferase in human tumor and corresponding normal tissue. Cancer Detect Prev 20:130-136

37. Rolhion C, Penault-Llorca F, Kemeny JL, Kwiatkowski F, Lemaire JJ, Chollet P, Finat-Duclos F, Verrelle P (1999) O(6)methylguanine-DNA methyltransferase gene (MGMT) expression in human glioblastomas in relation to patient characteristics and p53 accumulation. Int J Cancer 84:416-420

38. Siker ML, Chakravarti A, Mehta MP (2006) Should concomitant and adjuvant treatment with temozolomide be used as standard therapy in patients with anaplastic glioma? Crit Rev Oncol Hematol 60:99-111

39. Silber JR, Mueller BA, Ewers TG, Berger MS (1993) Comparison of O6-methylguanine-DNA methyltransferase activity in brain tumors and adjacent normal brain. Cancer Res 53:3416-3420

40. Stupp R, Mason WP, van den Bent MJ, Weller M, Fisher B, Taphoorn MJ, Belanger K, Brandes AA, Marosi C, Bogdahn U, Curschmann J, Janzer RC, Ludwin SK, Gorlia T, Allgeier A, Lacombe D, Cairncross JG, Eisenhauer E, Mirimanoff RO; European Organisation for Research, Treatment of Cancer Brain Tumor and Radiotherapy Groups; National Cancer Institute of Canada Clinical Trials Group (2005) Radiotherapy plus concomitant and adjuvant temozolomide for glioblastoma. N Engl J Med 352:987-996

41. Stupp R, Hegi ME (2007) Methylguanine methyltransferase testing in glioblastoma: when and how? J Clin Oncol 25:1459-1460

42. Tanaka S, Oka H, Fujii K, Watanabe K, Nagao K, Kakimoto A (2005) Quantitation of O6-methylguanine-DNA methyltransferase gene messenger RNA in gliomas by means of real-time RTPCR and clinical response to nitrosoureas. Cell Mol Neurobiol 25:1067-1071

43. Ueda S, Mineta T, Nakahara Y, Okamoto H, Shiraishi T, Tabuchi $\mathrm{K}$ (2004) Induction of the DNA repair gene O6-methylguanineDNA methyltransferase by dexamethasone in glioblastomas. J Neurosurg 101:659-663

44. Watanabe K, Tachibana O, Sata K, Yonekawa Y, Kleihues P, Ohgaki H (1996) Overexpression of the EGF receptor and p53 mutations are mutually exclusive in the evolution of primary and secondary glioblastomas. Brain Pathol 6:217-223

45. Watanabe T, Katayama Y, Komine C, Yoshino A, Ogino A, Ohta T, Fukushima T (2005) O6-methylguanine-DNA methyltransferase methylation and TP53 mutation in malignant astrocytomas and their relationships with clinical course. Int J Cancer 113:581-587

46. Watanabe T, Katayama Y, Yoshino A, Yachi K, Ohta T, Ogino A, Komine C, Fukushima T (2007) Aberrant hypermethylation of p14ARF and O6-methylguanine-DNA methyltransferase genes in astrocytoma progression. Brain Pathol 17:5-10

47. Wedge SR, Porteous JK, Glaser MG, Marcus K, Newlands ES (1997) In vitro evaluation of temozolomide combined with X-irradiation. Anticancer Drugs 8:92-97

48. Yuan Q, Matsumoto K, Nakabeppu Y, Iwaki T (2003) A comparative immunohistochemistry of O6-methylguanine-DNA methyltransferase and p53 in diffusely infiltrating astrocytomas. Neuropathology 23:203-209 